\title{
REPERCUSSÕES DA CIRURGIA BARIÁTRICA SOBRE PARÂMETROS METABÓLICOS. EXPERIÊNCIA DE 15 ANOS EM HOSPITAL DE MACEIÓ - BRASIL.
}

\author{
REPERCUSSIONS OF BARIATRIC SURGERY ON METABOLIC PARAMETERS: EXPERIENCE OF 15-YEAR FOLLOW-UP \\ IN A HOSPITAL IN MACEIÓ, BRAZIL
}
José Adailton PINHEIRO ${ }^{1 \oplus}$, Ingrid Ramalho Dantas de CASTRO ${ }^{2}{ }^{\circ}$, Ingrid Botelho RIBEIRO ${ }^{2 \odot}$, Marcus Vinícius Quirino FERREIRA ${ }^{3 \odot}$, Pérola Averbug FIREMAN ${ }^{1}{ }^{\circ}$, Marcos Antônio Duarte MADEIRO ${ }^{1 \odot}$, Ana Carolina Pastl PONTES ${ }^{1,2}$

\begin{abstract}
RESUMO - RACIONAL: A obesidade é caracterizada pelo acúmulo excessivo de gordura corporal prejudicial à saúde e nos últimos anos tem crescido significativamente na maioria dos países. A cirurgia bariátrica deverá ser recomendada para pacientes obesos que não obtiveram êxito no tratamento clínico e após análise da equipe multiprofissional. Objetivo: comparar os resultados metabólicos, perda ponderal e parâmetros associados à obesidade no pré e pós-operatório dos pacientes submetidos à cirurgia bariátrica. Métodos: estudo retrospectivo, descritivo, transversal, de abordagem quantitativa através da consulta a prontuários. Os dados foram coletados no período de maio a setembro de 2020, de indivíduos submetidos à cirurgia bariátrica no período de 15 anos (2003 a 2018). Foi realizada análise estatística comparativa e descritiva das variáveis antropométricas, metabólicas, bioquímicas e morbidades associadas. Resultados: a maioria era do sexo feminino (68,50\%). Em ambos os sexos a maior prevalência se encontravam na faixa etária de 30 a 39 anos e mais da metade tinha obesidade grau III. A técnica cirúrgica utilizada foi o gastroplastia em Y de Roux. Após 4 meses houve uma redução significativa do perfil lipídico, dos parâmetros antropométricos e enzimas hepáticas em ambos os sexos, os quais permaneceram em declínio no final do primeiro ano, com melhora acentuada da síndrome metabólica. Conclusões: $\mathrm{O}$ impacto positivo determinado pela gastroplastia na perda de peso, na redução do IMC e perfil lipídico é bastante relevante já após quatro meses, e se mantém após um ano da realização do procedimento, demonstrando benefícios na redução dos fatores de risco da síndrome metabólica.

DESCRITORES: Obesidade. Cirurgia Bariátrica. Síndrome Metabólica
\end{abstract}

ABSTRACT - BACKGROUND: Obesity is a disease characterized by an excessive accumulation of body fat, which is harmful to health, and it has grown significantly in the past years in the majority of countries. The surgery should be recommended to those patients with obesity who did not succeed in conservative clinical therapy after a rigorous analysis by a multidisciplinary team. Objective: The aim of this study was to compare metabolic results, weight loss, and parameters associated with obesity in the preoperative and postoperative periods of patients treated with bariatric surgery. Methods: This was a retrospective, descriptive, cross-sectional, and quantitative study through consultation medical records. Data were collected from May to September 2020 from individuals treated with bariatric surgery in a period of 15 years (from 2003 to 2018). A comparative and descriptive statistical analysis of anthropometric, metabolic, biochemical, and associated morbidities was performed. Results: The majority of patients were female (68.50\%). In both sexes, the highest prevalence was found in the age group of 30-39 years and more than half had grade III obesity. The surgical technique used was gastroplasty with Roux-en-Y gastric bypass. After 4 months, there was a significant reduction in the lipid profile, anthropometric parameters, and liver enzymes in both sexes, which remained decreasing till the end of the first year, with marked improvement in the metabolic syndrome (MS). Conclusions: The positive impact resulted from gastroplasty in terms of weight loss, reduction of body mass index, and lipid profile is quite relevant after 4 months and it is maintained until 1 year after the procedure, showing benefits in reducing the risk factors of the MS. HEADINGS: Obesity. Bariatric Surgery. Metabolic Syndrome

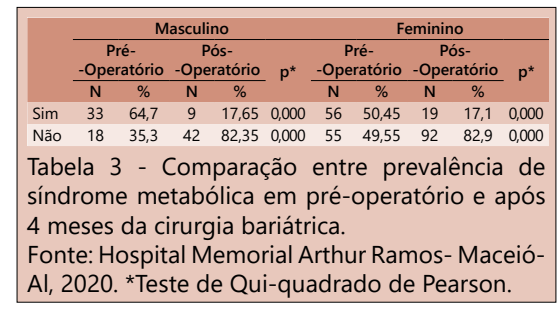

\section{Mensagem central}

O impacto positivo determinado pela gastroplastia na perda de peso, na redução do IMC, melhora dos parâmetros metabólicos e bioquímicos já é extremamente significativa após quatro meses, e se mantém ao fim do primeiro ano após o procedimento cirúrgico, mesmo nos pacientes que não se enquadram na categoria eutrófica.

\begin{tabular}{|l|}
\hline Perspectivas \\
\hline É importante a persistência das ações \\
nutricionais e comportamentais que irão \\
colaborar para a conquista e manutenção do \\
peso ideal e evitando reganhos de peso que os \\
façam retornar às situações de risco nas quais se \\
encontravam antes da gastroplastia. \\
\hline
\end{tabular}

Trabalho realizado no ${ }^{1}$ Hospital Memorial Arthur Ramos, Serviço de Cirurgia Bariátrica - Maceió - Alagoas - Brasil; ${ }^{2}$ Centro Universitário Tiradentes-UNIT/AL, Curso de Medicina - Maceió - Alagoas - Brasil; ${ }^{3}$ Centro Universitário CESMAC, Curso de Medicina - Maceió - Alagoas - Brasil

Como citar esse artigo: Pinheiro JA, Castro IRD, Ribeiro IB, Ferreira MVQ, Fireman PA, Madeiro MAD, Pontes ACP. Repercussões da cirurgia bariátrica sobre parâmetros metabólicos. Experiência de 15 anos em Hospital de Maceió - Brasil. ABCD Arq Bras Cir Dig. 2021;34(4):e1627. https://doi.org/10.1590/0102-672020210002e1627

Correspondência:

Ingrid Ramalho Dantas de Castro

Email: ingrid_ramalho1@hotmail.com
Fonte de financiamento: Não

Conflito de interesse: Não

Recebido: 02/07/2021

Aceito: 30/09/2021 


\section{INTRODUÇÃO}

A obesidade é uma doença caracterizada pelo acúmulo excessivo de gordura corporal prejudicial à saúde e nos últimos anos tem crescido significativamente na maioria dos países do mundo, sendo responsável por uma série de complicações que afetam diretamente a qualidade e a expectativa de vida desses indivíduos. ${ }^{19}$ A Organização Mundial da Saúde (OMS) define o diagnóstico através do índice de massa corporal (IMC) maior ou igual a $30 \mathrm{~kg} / \mathrm{m}^{14}$.

No Brasil, de acordo com o Ministério da Saúde, as doenças crônicas não transmissíveis foram responsáveis, em 2016, por 74\% do total de mortes, com destaque para doenças cardiovasculares (28\%), as neoplasias (18\%), as doenças respiratórias (6\%) e o diabetes (5\%). ${ }^{4}$ Durante o período de 2013-2018, a obesidade, excesso de peso e diabetes evoluíram intensamente.

A cirurgia bariátrica é indicada para pessoas com IMC acima de $40 \mathrm{~kg} / \mathrm{m}^{2}$ ou IMC a partir de $35 \mathrm{~kg} / \mathrm{m}^{2}$, mas que já apresentam determinadas patologias ocasionadas ou agravadas pela obesidade, de acordo com as recomendações da resolução do Conselho Federal de Medicina (CFM), N².131/2015, no qual ficaram estabelecidas vinte e uma comorbidades. Além dessas indicações é importante que exista falência do tratamento clínico da obesidade por, pelo menos, dois anos, contudo essa exigência não se aplica em indivíduos com IMC > 50kg/m².

No entanto, uma nova resolução do CFM ( $\left.n^{\circ} 2.172 / 2017\right)$, determinou que pacientes com IMC entre 30 e $34,9 \mathrm{~kg} / \mathrm{m}^{2}$, portadores de diabetes mellitus tipo 2 refratários ao tratamento clínico e atestado por dois endocrinologistas, podem realizar o procedimento, sendo denominado nessa situação de cirurgia metabólica, sendo a de primeiro escolha a gastroplastia em Y-de-Roux (BGYR). ${ }^{10}$

Essa intervenção promove a restrição alimentar através de mudanças estruturais no trato gastrointestinal. A sua realização vem crescendo nos dias atuais, sendo considerado um método eficaz no tratamento da obesidade mórbida e no controle de peso em longo prazo, mas tem ganhado importância também no controle da diabetes, na redução dos fatores de risco cardiovasculares e de outras comorbidades relacionadas à obesidade e da síndrome metabólica nos indivíduos refratários ao tratamento clínico. ${ }^{7,13,16}$

A cirurgia deverá ser recomendada para aqueles pacientes obesos que não obtiveram êxito no tratamento clínico conservador (dieta e medicamentos) e após rigorosa análise da equipe multiprofissional, com avaliação do estado nutricional, das condições e do perfil psicológico e deverá seguir as recomendações médicas e nutricionais após o procedimento, complementado com um plano alimentar de forma correta e individualizada, com suplementação nutricional, além de manter atividade física. Isto porque o sucesso do tratamento cirúrgico depende essencialmente do equilíbrio emocional e das mudanças nos hábitos alimentares e estilo de vida do indivíduo. ${ }^{8,21}$

Desde o início do tratamento o paciente deve ter ciência do diagnóstico da sua doença e ser orientado dos riscos, benefícios e consequências da cirurgia. Assim, o objetivo deste estudo foi comparar os resultados metabólicos, perda ponderal e parâmetros associados à obesidade no pré e pós-operatório dos pacientes submetidos à cirurgia bariátrica.

\section{MÉTODOS}

Trata-se de um estudo retrospectivo, descritivo, do tipo transversal, de abordagem quantitativa, no ambulatório de clínica médica do Hospital Memorial Arthur Ramos que realiza, em média, oito cirurgias bariátricas por mês, pelo mesmo profissional médico e nutricionista, localizado no município de Maceió - AL.
Os dados foram coletados no período de maio a setembro de 2020, a partir de consulta a prontuários de indivíduos submetidos à cirurgia bariátrica no período de 15 anos, de 2003 a 2018.

Foi estabelecido como critério de inclusão para o estudo todo paciente do qual o prontuário contivesse os dados completos de 4 meses antes e 4 meses após a cirurgia. Desse modo, foram selecionados 162 pacientes. Entretanto destes, apenas 96 pacientes continham dados após 1 ano da cirurgia, sendo os mesmos utilizados para análise da evolução.

Portanto foi analisado um protocolo nutricional padronizado constituído pelos seguintes itens: a) dados sociodemográficos b) parâmetros antropométricos (peso, estatura, circunferência abdominal, índice de massa corporal - IMC); c) parâmetros bioquímicos: hemoglobina, hematócrito, proteínas totais, albumina, ferro sérico, ferritina, ácido fólico, cálcio, vitamina B12, outras vitaminas e transaminases (TGO, TGP), fosfatase alcalina,-GGT (gama-gt), d) parâmetros metabólicos (colesterol total, colesterol LDL e HDL, triglicérides, glicemia de jejum, hemoglobina glicada) que foram solicitados pelo cirurgião e outros médicos da equipe. Essas variáveis coletadas foram referentes ao período pré e pós-operatório. Foi identificada a presença de morbidades associadas (diabetes mellitus - DM, hipertensão arterial sistêmica - HAS, dislipidemia, doença do refluxo gastroesofágico - DRGE e esteatose hepática), no período pré-operatório.

A fim de classificar a obesidade, de acordo com o IMC, foram aplicados os critérios recomendados pela Organização Mundial de Saúde e pela Associação Brasileira para Estudo da Obesidade e da Síndrome metabólica1. Sendo o estado nutricional classificado em: baixo peso (IMC $<18,5 \mathrm{~kg} / \mathrm{m}^{2}$ ), eutrófico (IMC entre 18,5 e $24,9 \mathrm{~kg} / \mathrm{m}^{2}$ ), sobrepeso (IMC entre 25 e $29,9 \mathrm{~kg} / \mathrm{m}^{2}$ ), obesidade grau I (IMC= 30 a $\left.34,9 \mathrm{~kg} / \mathrm{m}^{2}\right)$, grau II (IMC= 35 a 39,9 $\left.\mathrm{kg} / \mathrm{m}^{2}\right)$ e grau III (IMC $\left.>40 \mathrm{~kg} / \mathrm{m}^{2}\right)$.

Para o reconhecimento da presença da síndrome metabólica (SM), foi aplicada a recomendação da Sociedade Brasileira de Cardiologia, que contempla os parâmetros da NCEP-ATP III, definida com 3 ou mais critérios: obesidade central $(>102 \mathrm{~cm}$ para homens e $>88 \mathrm{~cm}$ para mulheres); $\mathrm{HDL}$-colesterol $(<40 \mathrm{mg} / \mathrm{dl}$ para homens e $<50 \mathrm{mg} / \mathrm{dl}$ para mulheres); Triglicerídeos $=150 \mathrm{mg} / \mathrm{dl}$ (ou tratamento para hipertrigliceridemia); PA (PAS $=130 \mathrm{mmHg}$ e/ou PAD $=85 \mathrm{mmHg}$ ) ou tratamento para hipertensão arterial e Glicemia $=100 \mathrm{mg} / \mathrm{dl}$ (ou tratamento para DM) ${ }^{22}$.

Os dados foram inseridos no programa Microsoft Excel 2010(r). Para todos os testes estatísticos a significância foi determinada quando $\mathrm{p} £ 0,05$. A análise descritiva foi apresentada em frequência absoluta e percentual. Foi utilizado o teste " $t$ " pareado para comparar a média das variáveis antropométricas, bioquímicas e metabólicas no período pré e pós-operatório, Para medir a associação entre as variáveis da síndrome metabólica no pré e pós-operatório, foi utilizado o teste de $x^{2}$ de Pearson.

A elaboração do estudo ocorreu conforme as normas éticas baseadas na resolução 466/12 do Conselho Nacional de Saúde e o projeto de pesquisa foi aprovado pelo Comitê de Ética em Pesquisa (CEP) do Centro Universitário TiradentesUNIT-AL (parecer n 4.014.670).

\section{RESULTADOS}

Dos 162 pacientes analisados, 111 eram do sexo feminino e 51 do sexo masculino. Em ambos os sexos a maior prevalência se encontravam na faixa etária de 30 a 39 anos sendo $41,50 \%$ nas mulheres e 39,20\% nos homens (Tabela 1). A técnica cirúrgica utilizada foi o gastroplastia em $Y$ de Roux (bypass gástrico).

Na tabela 2, observa-se que tanto entre os homens, quanto entre as mulheres mais da metade possuíam HAS, dislipidemia, com destaque para a esteatose hepática representando $73,45 \%$ do 
total de pacientes, antes da realização da cirurgia. Enquanto DM e DRGE foram observadas em menos da metade.

Foi constatada uma redução da síndrome metabólica em $37 \%$ dos pacientes de ambos os sexos em apenas 4 meses da cirurgia. (Tabela 3).

A tabela 4 apresenta informações relacionadas ao grau de obesidade. Percebe-se que anteriormente a cirurgia a obesidade grau III representava $64,20 \%$ do número total de pacientes, sendo $86,25 \%$ homens e $54,50 \%$ mulheres. No sexo masculino, após 4 meses da cirurgia, $47 \%$ passou à classe de obesidade grau I, evoluindo com diminuição do IMC, dessa forma $68,95 \%$ alcançou em 1 ano a classe de sobrepeso e 10,35\% eutrófico. Entre as mulheres, no período de 4 meses pós-operatório, $43,3 \%$ passou para obesidade grau I e posteriormente com 1 ano, $52,25 \%$ passou para classe de sobrepeso e $31,35 \%$ atingiram a categoria eutrófica.

Tabela 1 - Faixa etária dos pacientes submetidos à cirurgia bariátrica.

\begin{tabular}{|c|c|c|c|c|c|c|}
\hline \multirow{2}{*}{ Idade } & \multicolumn{2}{|c|}{ Masculino } & \multicolumn{2}{|c|}{ Feminino } & \multicolumn{2}{|c|}{ Total } \\
\hline & $\mathrm{N}$ & $\%$ & $N$ & $\%$ & $\mathrm{~N}$ & $\%$ \\
\hline 18 a 29 anos & 15 & 29,4 & 22 & 19,8 & 37 & 22,8 \\
\hline 30 a 39 anos & 20 & 39,2 & 46 & 41,5 & 66 & 40,75 \\
\hline 40 a 49 anos & 10 & 19,6 & 22 & 19,8 & 32 & 19,75 \\
\hline 50 a 59 anos & 4 & 7,9 & 16 & 14,4 & 20 & 12,35 \\
\hline$=60$ anos & 2 & 3,9 & 5 & 4,5 & 7 & 4,35 \\
\hline
\end{tabular}

Fonte: Hospital Memorial Arthur Ramos- Maceió-Al, 2020.

Tabela 2 - Prevalência de morbidades associadas à obesidade em período pré-operatório de cirurgia bariátrica.

\begin{tabular}{|c|c|c|c|c|c|c|}
\hline \multirow[t]{2}{*}{ Morbidades Associada } & \multicolumn{2}{|c|}{$\begin{array}{c}\text { Masculino } \\
\text { Pré-Operatório }\end{array}$} & \multicolumn{2}{|c|}{$\begin{array}{c}\text { Feminino } \\
\text { Pré-Operatório }\end{array}$} & \multicolumn{2}{|c|}{ Total } \\
\hline & $\mathrm{N}$ & $\%$ & $\mathrm{~N}$ & $\%$ & $\mathrm{~N}$ & $\%$ \\
\hline \multicolumn{7}{|l|}{ Hipertensão Arterial } \\
\hline Sim & 29 & 56,86 & 58 & 52,25 & 87 & 53,7 \\
\hline Não & 22 & 43,14 & 53 & 47,75 & 75 & 46,3 \\
\hline \multicolumn{7}{|l|}{ Diabetes Mellitus } \\
\hline Sim & 17 & 33,33 & 24 & 21,6 & 41 & 25,3 \\
\hline Não & 34 & 66,66 & 87 & 78,4 & 121 & 74,7 \\
\hline \multicolumn{7}{|l|}{ Esteatose Hepática } \\
\hline Sim & 41 & 80,4 & 78 & 70,3 & 119 & 73,45 \\
\hline Não & 10 & 19,6 & 33 & 29,7 & 43 & 26,55 \\
\hline \multicolumn{7}{|l|}{ Dislipidemia } \\
\hline Sim & 33 & 64,7 & 70 & 63 & 103 & 63,6 \\
\hline Não & 18 & 35,3 & 41 & 37 & 59 & 36,4 \\
\hline \multicolumn{7}{|l|}{ DRGE } \\
\hline Sim & 18 & 35,3 & 38 & 34,25 & 56 & 34,55 \\
\hline Não & 33 & 64,7 & 73 & 65,75 & 106 & 65,45 \\
\hline
\end{tabular}

Fonte: Hospital Memorial Arthur Ramos- Maceió-Al, 2020.

Tabela 3 - Comparação entre prevalência de síndrome metabólica em pré-operatório e após 4 meses da cirurgia bariátrica.

\begin{tabular}{|c|c|c|c|c|c|c|c|c|c|c|}
\hline & \multicolumn{5}{|c|}{ Masculino } & \multicolumn{5}{|c|}{ Feminino } \\
\hline & \multicolumn{2}{|c|}{ Pré-Operatório } & \multicolumn{2}{|c|}{ Pós-Operatório } & \multirow{2}{*}{$\mathrm{p}^{*}$} & \multicolumn{2}{|c|}{ Pré-Operatório } & \multicolumn{2}{|c|}{ Pós-Operatório } & \multirow[b]{2}{*}{$\mathrm{p}^{*}$} \\
\hline & $\mathrm{N}$ & $\%$ & $\mathrm{~N}$ & $\%$ & & $\mathrm{~N}$ & $\%$ & $\mathrm{~N}$ & $\%$ & \\
\hline Sim & 33 & 64,7 & 9 & 17,65 & 0,000 & 56 & 50,45 & 19 & 17,1 & 0,000 \\
\hline Não & 18 & 35,3 & 42 & 82,35 & 0,000 & 55 & 49,55 & 92 & 82,9 & 0,000 \\
\hline
\end{tabular}

Fonte: Hospital Memorial Arthur Ramos- Maceió-Al, 2020. *Teste de Qui-quadrado de Pearson.

Tabela 4 - Comparação do estado nutricional no período pré e pós-operatório de cirurgia bariátrica no sexo masculino e feminino.

\begin{tabular}{|c|c|c|c|c|c|c|}
\hline \multirow{2}{*}{ Masculina } & \multicolumn{2}{|c|}{ Pré-Operatório } & \multicolumn{2}{|c|}{ Pós-Operatório 4 meses } & \multicolumn{2}{|c|}{ Pós-Operatório 1 ano } \\
\hline & $\mathrm{N}$ & $\%$ & $\mathrm{~N}$ & $\%$ & $\mathrm{~N}$ & $\%$ \\
\hline Eutrófico & 0 & 0 & 0 & 0 & 3 & 10,35 \\
\hline Sobrepeso & 0 & 0 & 17 & 33,4 & 20 & 68,95 \\
\hline Obeso Grau I & 0 & 0 & 24 & 47 & 6 & 20,7 \\
\hline Obeso Grau II & 7 & 13,75 & 7 & 13,7 & 0 & 0 \\
\hline Obeso Grau III & 44 & 86,25 & 3 & 5,9 & 0 & 0 \\
\hline \multirow{2}{*}{ Feminina } & \multicolumn{2}{|c|}{ Pré-Operatório } & \multicolumn{2}{|c|}{ Pós-Operatório 4 meses } & \multicolumn{2}{|c|}{ Pós-operatório 1 ano } \\
\hline & $\mathrm{N}$ & $\%$ & $\mathrm{~N}$ & $\%$ & $\mathrm{~N}$ & $\%$ \\
\hline Eutrófico & 0 & 0 & 0 & 0 & 21 & 31,35 \\
\hline Sobrepeso & 0 & 0 & 36 & 32,4 & 35 & 52,25 \\
\hline Obeso Grau I & 2 & 1,8 & 48 & 43,3 & 9 & 13,4 \\
\hline Obeso Grau II & 49 & 44,15 & 22 & 19,8 & 2 & 3 \\
\hline Obeso Grau III & 60 & 54,5 & 5 & 4,5 & 0 & 0 \\
\hline
\end{tabular}

Fonte: Hospital Memorial Arthur Ramos- Maceió-Al, 2020 
A comparação dos resultados entre as médias do peso total e IMC dos pacientes, no período pré e pós-operatório, retratam uma redução, com valor expressivo em ambos os sexos, e continua em declínio até 1 ano. (Tabelas 5 e 6)

Na avaliação dos parâmetros metabólicos, houve redução do nível de HDL de ambos os sexos após 4 meses da cirurgia (Tabela 5), porém, com elevação ao fim do primeiro ano (Tabela 6).
Os valores de $\mathrm{LDL}$, colesterol total, triglicérides e glicose em jejum demonstraram redução significativa em ambos os sexos após 4 meses (Tabela 5). Quanto à hemoglobina glicada, já se observa uma redução no quarto mês (Tabela 5), sendo mais relevante após 1 ano. (Tabela 6)

Os valores dos parâmetros bioquímicos após 4 meses da intervenção TGO, TGP e GGT apresentaram redução significativa

Tabela 5 - Comparação dos parâmetros antropométricos, metabólicos e bioquímicos de pessoas obesas no período pré e 4 meses após a cirurgia bariátrica.

\begin{tabular}{|c|c|c|c|c|c|c|}
\hline & \multicolumn{3}{|c|}{ Masculino } & \multicolumn{3}{|c|}{ Feminino } \\
\hline & $\begin{array}{c}\text { Pré-Operatório } \\
\text { Média } \pm \text { DP }\end{array}$ & $\begin{array}{c}\text { Pós-Operatório } \\
\text { Média } \pm \text { DP }\end{array}$ & $\mathrm{p}^{*}$ & $\begin{array}{c}\text { Pré-Operatório } \\
\text { Média } \pm \text { DP }\end{array}$ & $\begin{array}{c}\text { Pós-Operatório } \\
\text { Média } \pm \text { DP }\end{array}$ & $p^{*}$ \\
\hline \multicolumn{7}{|l|}{ Parâmetros Antropométricos } \\
\hline Peso (kg) & $133,87 \pm 22,25$ & $97,36 \pm 16,72$ & 0,000 & $107,75 \pm 13,41$ & $84,39 \pm 11,50$ & 0,000 \\
\hline IMC $\left(\mathrm{kg} / \mathrm{m}^{2}\right)$ & $44,57 \pm 5,12$ & $32,53 \pm 4,46$ & 0,000 & $41,59 \pm 4,70$ & $35,57 \pm 4,35$ & 0,000 \\
\hline \multicolumn{7}{|l|}{ Parâmetros Metabólicos } \\
\hline $\mathrm{HDL}(\mathrm{mlg} / \mathrm{dl})$ & $41,98 \pm 10,12$ & $39,18 \pm 10,26$ & 0,064 & $49,67 \pm 10,79$ & $44,88 \pm 9,45$ & 0,000 \\
\hline LDL (mlg/dl) & $114,49 \pm 29,55$ & $94,58 \pm 33,37$ & 0,001 & $125,28 \pm 30,14$ & $99,37 \pm 25,80$ & 0,000 \\
\hline $\mathrm{CT}(\mathrm{mlg} / \mathrm{dl})$ & $188,85 \pm 31,65$ & $154,30 \pm 45,92$ & 0,000 & $203,17 \pm 40,88$ & $162,53 \pm 30,42$ & 0,000 \\
\hline $\mathrm{TG}(\mathrm{mlg} / \mathrm{dl})$ & $184,08 \pm 86,18$ & $99,51 \pm 40,28$ & 0,000 & $149,65 \pm 80,02$ & $104,40 \pm 43,50$ & 0,000 \\
\hline Glicose em Jejum (mlg/dl) & $107,89 \pm 35,48$ & $81,63 \pm 8,78$ & 0,000 & $95,10 \pm 27,83$ & $84,06 \pm 24,86$ & 0,000 \\
\hline Hemoglobina glicada (\%) & $8,07 \pm 10,15$ & $6,63 \pm 7,52$ & 0,568 & $6,09 \pm 1,18$ & $5,47 \pm 0,90$ & 0,000 \\
\hline \multicolumn{7}{|l|}{ Parâmetros Bioquímicos } \\
\hline Hemoglobina (g/dl) & $15 \pm 1,34$ & $14,26 \pm 1,07$ & 0,000 & $12,87 \pm 0,96$ & $12,52 \pm 0,95$ & 0,004 \\
\hline Hematócrito (\%) & $44,44 \pm 5,59$ & $42,91 \pm 3,24$ & 0,053 & $39,03 \pm 3,76$ & $38,12 \pm 2,77$ & 0,024 \\
\hline Proteínas totais(g/dl) & $7,25 \pm 0,72$ & $8,82 \pm 11,53$ & 0,013 & $7,12 \pm 0,54$ & $6,76 \pm 0,38$ & 0,000 \\
\hline Albumina $(\mathrm{g} / \mathrm{dl})$ & $4,28 \pm 0,45$ & $12,37 \pm 52,34$ & 0,325 & $3,99 \pm 0,41$ & $4,01 \pm 0,38$ & 0,406 \\
\hline Ferro sérico (ug/dl) & $99,65 \pm 33,22$ & $87,10 \pm 30,80$ & 0,448 & $80,8 \pm 34,19$ & $70,13 \pm 28,02$ & 0,020 \\
\hline Ferritina (ng/ml) & $351,33 \pm 286,97$ & $251,11 \pm 136,82$ & 0,010 & $102,50 \pm 247,62$ & $90,06 \pm 90,61$ & 0,068 \\
\hline Vit. B12(pg/ml) & $395,43 \pm 126,31$ & $394,55 \pm 152,35$ & 0,686 & $365,72 \pm 151,63$ & $383,22 \pm 159,53$ & 0,233 \\
\hline Ácido Fólico (ng/ml) & $11,30 \pm 5,84$ & $15,09 \pm 8,91$ & 0,028 & $10,62 \pm 5,16$ & $17,46 \pm 5,60$ & 0,000 \\
\hline TGO (U/I) & $34,47 \pm 23,04$ & $23,63 \pm 6,02$ & 0,002 & $25,96 \pm 15,86$ & $30,12 \pm 20,56$ & 0,017 \\
\hline TGP $(U / \mathrm{I})$ & $49,75 \pm 23,92$ & $33,01 \pm 16,70$ & 0,000 & $31,46 \pm 32,01$ & $33,62 \pm 26,44$ & 0,048 \\
\hline Fosf. Alcalina (U/I) & $68,16 \pm 23,48$ & $49,96 \pm 25,19$ & 0,920 & $76,01 \pm 29,46$ & $118 \pm 66,92$ & 0,172 \\
\hline Gama GT (U/I) & $55,22 \pm 26,81$ & $24,15 \pm 12,63$ & 0,000 & $39,68 \pm 34,25$ & $25,72 \pm 27,18$ & 0,000 \\
\hline Cálcio (mg/dL) & $11,07 \pm 11,26$ & $9,52 \pm 0,59$ & 0,362 & $10,10 \pm 8,42$ & $9,89 \pm 6,58$ & 0,853 \\
\hline
\end{tabular}

Fonte: Hospital Memorial Arthur Ramos- Maceió-Al, 2020.

Tabela 6 - Comparação dos parâmetros antropométricos, metabólicos e bioquímicos de pessoas obesas no período pré e 1 ano após a cirurgia bariátrica.

\begin{tabular}{|c|c|c|c|c|c|c|}
\hline & \multicolumn{3}{|c|}{ Masculino } & \multicolumn{3}{|c|}{ Feminino } \\
\hline & Pré-Operatório & Pós-Operatório & \multirow{2}{*}{$p^{*}$} & Pré-Operatório & Pós-Operatório & \multirow{2}{*}{$p^{*}$} \\
\hline & Média \pm DP & Média \pm DP & & Média \pm DP & Média \pm DP & \\
\hline \multicolumn{7}{|l|}{ Parâmetros Antropométricos } \\
\hline Peso (kg) & $133,87 \pm 22,25$ & $81,11 \pm 9,60$ & 0,000 & $107,75 \pm 13,41$ & $70,71 \pm 9,76$ & 0,000 \\
\hline IMC $\left(\mathrm{kg} / \mathrm{m}^{2}\right)$ & $44,57 \pm 5,12$ & $26,98 \pm 5,64$ & 0,000 & $41,59 \pm 4,70$ & $27,29 \pm 3,52$ & 0,000 \\
\hline \multicolumn{7}{|l|}{ Parâmetros Metabólicos } \\
\hline $\mathrm{HDL}(\mathrm{mlg} / \mathrm{dl})$ & $41,98 \pm 10,12$ & $51,66 \pm 12,25$ & 0,324 & $49,67 \pm 10,79$ & $53,55 \pm 12,09$ & 0,028 \\
\hline LDL (mlg/dl) & $114,49 \pm 29,55$ & $80,99 \pm 22,71$ & 0,001 & $125,28 \pm 30,14$ & $99,11 \pm 79,43$ & 0,023 \\
\hline CT (mlg/dl) & $188,85 \pm 31,65$ & $149,20 \pm 25,78$ & 0,001 & $203,17 \pm 40,88$ & $155,04 \pm 34,32$ & 0,000 \\
\hline $\mathrm{TG}(\mathrm{mlg} / \mathrm{dl})$ & $184,08 \pm 86,18$ & $76,36 \pm 24,00$ & 0,000 & $149,65 \pm 80,02$ & $87,35 \pm 13,08$ & 0,000 \\
\hline Glicose em Jejum (mlg/dl) & $107,89 \pm 35,48$ & $80,20 \pm 8,25$ & 0,007 & $95,10 \pm 27,83$ & $84,03 \pm 8,66$ & 0,001 \\
\hline Hemoglobina glicada (\%) & $8,07 \pm 10,15$ & $4,77 \pm 0,56$ & 0,009 & $6,09 \pm 1,18$ & $5,30 \pm 0,54$ & 0,001 \\
\hline \multicolumn{7}{|l|}{ Parâmetros Bioquímicos } \\
\hline Hemoglobina (g/dl) & $15 \pm 1,34$ & $13,75 \pm 1,28$ & 0,031 & $12,87 \pm 0,96$ & $12,01 \pm 1,00$ & 0,005 \\
\hline Hematócrito (\%) & $44,44 \pm 5,59$ & $41,29 \pm 3,71$ & 0,022 & $39,03 \pm 3,76$ & $36,78 \pm 2,95$ & 0,001 \\
\hline Proteínas totais(g/dl) & $7,25 \pm 0,72$ & $6,85 \pm 0,51$ & 0,046 & $7,12 \pm 0,54$ & $6,4 \pm 0,82$ & 0,015 \\
\hline Albumina (g/dl) & $4,28 \pm 0,45$ & $4,22 \pm 0,28$ & 0,298 & $3,99 \pm 0,41$ & $4,04 \pm 0,34$ & 0,394 \\
\hline Ferro sérico (ug/dl) & $99,65 \pm 33,22$ & $87,09 \pm 21,37$ & 0,059 & $80,8 \pm 34,19$ & $83,55 \pm 32,47$ & 0,484 \\
\hline Ferritina (ng/ml) & $351,33 \pm 286,97$ & $208,37 \pm 112,18$ & 0,092 & $102,50 \pm 247,62$ & $101,52 \pm 105,98$ & 0,351 \\
\hline Vit. B12(pg/ml) & $395,43 \pm 126,31$ & $472,75 \pm 248,09$ & 0,667 & $365,72 \pm 151,63$ & $293,40 \pm 113,83$ & 0,028 \\
\hline Ácido Fólico (ng/ml) & $11,30 \pm 5,84$ & $17,32 \pm 6,09$ & 0,002 & $10,62 \pm 5,16$ & $26,29 \pm 36,89$ & 0,037 \\
\hline TGO (U/I) & $34,47 \pm 23,04$ & $29,80 \pm 13,68$ & 0,499 & $25,96 \pm 15,86$ & $27,37 \pm 13,08$ & 0,586 \\
\hline TGP (U/I) & $49,75 \pm 23,92$ & $32,62 \pm 17,29$ & 0,060 & $31,46 \pm 32,01$ & $31,46 \pm 32,02$ & 0,631 \\
\hline Gama GT (U/I) & $55,22 \pm 26,81$ & $27,28 \pm 12,79$ & 0,000 & $39,68 \pm 34,25$ & $19,83 \pm 10,26$ & 0,001 \\
\hline Cálcio (mg/dL) & $11,07 \pm 11,26$ & $8,89 \pm 2,27$ & 0,868 & $10,10 \pm 8,42$ & $12,00 \pm 12,71$ & 0,214 \\
\hline
\end{tabular}

Fonte: Hospital Memorial Arthur Ramos- Maceió-Al, 2020. 
em ambos os sexos. As proteínas totais tiveram um pequeno aumento nos homens nos primeiros 4 meses e uma redução insignificante após 1 ano. Nas mulheres houve pouca redução nos dois períodos, porém, mantiveram-se dentro dos parâmetros da normalidade em ambos os sexos. Hematócrito e hemoglobina não apresentaram reduções relevantes nos valores após 4 meses, já a ferritina houve uma maior redução.

Em relação a vitamina B12 praticamente não ocorreu redução no quarto mês, porém após 1 ano ocorreu apenas no sexo feminino. Quanto ao ácido fólico houve aumento durante todo o tempo analisado em ambos os sexos (Tabelas 5 e 6).

\section{DISCUSSÃO}

Segundo o Instituto Brasileiro de Geografia e Estatística (IBGE), a obesidade mais que dobrou no país na população com 20 anos ou mais em 2019, de acordo com a Pesquisa Nacional de Saúde, a obesidade entre as mulheres na mesma faixa etária passou de $14,5 \%$ para $30,2 \%$ e se manteve acima da masculina, que subiu de $9,6 \%$ para $22,8 \%{ }^{14}$. A maioria dos pacientes submetidos a cirurgia bariátrica nesse estudo foi do sexo feminino $(68,50 \%)$, corroborando os achados de diversas pesquisas nacionais e internacionais, com maior prevalência na faixa etária de 30 a 39 anos em ambos os sexos 6,21,26.

Uma metanálise de 89 estudos realizados em outros países sobre o impacto da gastroplastia no tratamento da obesidade demonstrou que a média de idade dos pacientes é de 38 anos, e que mais de um terço deles é do sexo feminino ${ }^{26}$. Outra metanálise envolvendo 134 estudos e um total de 22.094 pacientes demonstrou que $73 \%$ são do sexo feminino, com média de idade de 39 anos $^{6}$.

Além dos dados epidemiológicos, que comprovam um crescimento e prevalência da obesidade em mulheres, alguns estudos apontam também para outros fatores que poderiam explicar o predomínio de mulheres em relação à cirurgia bariátrica, como motivações estéticas pessoais e estigma da sociedade que cultua um padrão de beleza de mulheres magras. Diferentemente das mulheres, os homens tendem a buscar esse recurso apenas quando ocorre comprometimento da saúde e de suas atividades físicas cotidianas ${ }^{8}$.

Ao avaliar o impacto da perda de peso após a cirurgia bariátrica, percebe-se que nesta pesquisa, assim como em diversos estudos, esse procedimento vem se mostrando bastante eficaz ${ }^{3,11,12}$

Quanto aos dados de antropometria, os resultados aqui apresentados demonstram redução média de $36,51 \mathrm{~kg}$ no peso corporal e de $12,04 \mathrm{~kg} / \mathrm{m} 2$ no IMC no sexo masculino no período de 4 meses pós gastroplastia, já nas mulheres houve uma redução menos significativa com média de $23,36 \mathrm{~kg}$, e de $6,02 \mathrm{~kg} / \mathrm{m} 2$ no IMC neste mesmo período. Após 1 ano da cirurgia a diminuição do peso corporal média foi de $52,76 \mathrm{~kg}$ e do IMC foi de $17,59 \mathrm{~kg} / \mathrm{m} 2$ no sexo masculino, e no sexo feminino uma redução de $37,04 \mathrm{~kg}, 14,3 \mathrm{~kg} / \mathrm{m}^{2}$ no IMC. Com $31,35 \%$ das mulheres e 10,35\% dos homens alcançando a categoria eutrófica e mais da metade alcançando sobrepeso em ambos o sexo.

Estes dados implicam em expressiva redução, a partir do quarto mês, tanto na mortalidade cardiovascular como em outras doenças associadas ao excesso de peso. Salientamos que esse resultado corrobora com outros trabalhos científicos os quais identificaram resultados positivos precoces após gastroplastia, reduzindo significativamente o IMC 23,24.

Na avaliação das morbidades pré-operatórias, a presente pesquisa demonstrou que 104 de 111 pacientes do sexo feminino $(93,69 \%)$ e 48 de 51 pacientes do sexo masculino (94,11\%) possuíram algum tipo, indicando, desse modo, que a obesidade é uma condição clínica que predispõe surgimento de outras doenças. As morbidades mais prevalentes nos homens e mulheres, respectivamente, foram esteatose hepática $(80,4 \%$ e 70,3\%), dislipidemias (64,7\% e 63\%), síndrome metabólica (64,7\% e 54,45\%), hipertensão arterial (56,86\% e 52,25\%), DRGE (35,3\% e 34,25\%) e por último diabetes mellitus com (33,33\% e $21,6 \%)$. Esses dados também foram encontrados em outros estudos ${ }^{3,8}$.

Sabe-se que o "bypass" duodenal e jejunal, realizado no procedimento, reduz progressivamente a glicemia de jejum e melhora a resistência insulínica. Essa intervenção passou a ser também denominada cirurgia metabólica, pelas evidências que os diversos estudos têm demonstrado em relação a eficácia do tratamento da diabetes mellitus e a redução significativa da SM em ambos os sexos no final do primeiro ano pós-procedimento, o que também foi evidenciado nesta pesquisa ${ }^{2,18}$.

Estudos recentes têm interrogado o mecanismo pelo qual pacientes obesos mórbidos controlam o DM2 após cirurgias bariátricas. Na realidade, a perda de peso é parcialmente responsável pela reversão do DM2. A derivação intestinal que é realizada nessas operações provoca um efeito imediato de melhora e um aumento da produção de insulina, devido à produção acentuada de (GLP-1) glucagon-like peptide- 1 eà ação melhorada do (GIP) gastric inhibitory polypeptide, ou glucosedependent insulinotropic polypeptide, que contribuem para a melhora dos níveis glicêmicos e, consequentemente, do DM2 $2^{25}$.

Dessa forma, a redução relevante do peso corporal e da gordura visceral, alcançada com a gastroplastia determina, já em curto prazo, impacto positivo sobre o controle/resolução das comorbidades associadas à obesidade, incluindo SM, DM, dislipidemia, HAS, esteatose hepática e doenças cardiovasculares, com consequente aumento da expectativa de vida. Determinando a cirurgia bariátrica como sendo, na atualidade, o tratamento de escolha para obesos graves nos quais a terapia medicamentosacomportamental não foi eficaz ${ }^{15}$.

Por outro lado, é comum ocorrer desnutrição e déficit de nutrientes após esse procedimento. No BGYR há maior prevalência de deficiência de vitamina B12, ferro e ácido fólico ${ }^{5}$. As deficiências ocorrem, geralmente, devido restrição na ingestão alimentar, pelo impacto fisiológico das mudanças anatômicas, assim como pela intolerância alimentar e pela não adesão ao tratamento com o uso de polivitamínicos.

Considerando esse fato, os pacientes submetidos à cirurgia bariátrica em nosso estudo, realizaram acompanhamento regular clínico e nutricional para detectar possíveis deficiências nutricionais e foram realizadas suplementações vitamínicas e minerais, consequentemente não foi observada redução expressiva desses nutrientes.

A deficiência da vitamina B12 pode ocorrer em consequência de vários fatores como ocorre redução na produção gástrica de ácido clorídrico, não há a conversão de pepsinogênio em pepsina, a qual é necessária para a liberação da vitamina B12 presente em alimentos protéicos. Além disso, o fator intrínseco é produzido pelas células parietais do estômago. Quando não há a produção de fator intrínseco ou esta é insuficiente, como acontece com a redução do compartimento gástrico, não há absorção de vitamina B12 no íleo distal, dando origem a anemia perniciosa ${ }^{5}$. Os baixos níveis de vitamina B12 podem ser visualizados após seis meses de pós- operatório, porém na maioria das vezes ocorre após um ano ou mais quando seu armazenamento no fígado encontra-se esgotado, o que foi evidenciado em nosso estudo no sexo feminino ${ }^{5}$.

A dislipidemia, presente na maioria dos pacientes de ambos os sexos deste estudo, teve redução significativa do CT, LDL e TG ao longo do primeiro ano de pós-operatório. Em relação ao HDL houve aumento apenas após o $4^{\circ}$ mês de pós-operatório.

Finalmente, os estudos demonstram de forma inquestionável, a eficácia da gastroplastia no tratamento da obesidade ${ }^{17,24}$. 
É conhecido que o nível de triglicerídeos é reduzido de forma consistente imediatamente após a cirurgia e é mantido a longo prazo, mesmo que o paciente tenha reganho de peso ${ }^{20}$. Reduções de maior magnitude são observadas após a realização da cirurgia de by-pass gástrico, que além de ser restritiva, é disabsortiva ${ }^{24}$. Sendo consequência da mudança nos hábitos alimentares, caracterizados pela ingestão de alimentos em pequenas quantidades, com menor teor calórico e que propicia adoção de hábitos de vida saudáveis ${ }^{21}$.

\section{CONCLUSÃO}

Esses dados corroboram a importância, da persistência das ações nutricionais e comportamentais que irão colaborar para a conquista e manutenção do peso ideal e evitando reganhos de peso que os façam retornar às situações de risco nas quais se encontravam antes da gastroplastia.

Concluímos que o impacto positivo determinado pela gastroplastia na perda de peso, na redução do IMC, melhora dos parâmetros metabólicos e bioquímicos já é extremamente significativa após quatro meses, e se mantém ao fim do primeiro ano após o procedimento cirúrgico, mesmo nos pacientes que não se enquadram na categoria eutrófica.

\section{REFERÊNCIAS}

1. Associação Brasileira para o Estudo da Obesidade e da Síndrome Metabólica. Diretrizes brasileiras de obesidade 2016 / ABESO Associação Brasileira para o Estudo da Obesidade e da Síndrome Metabólica. - 4.ed. - São Paulo, SP

2. Ayoub JAS, Alonso PA, Guimarães LMV. Effects of bariatric surgery on the metabolic syndrome. Arq Bras Cir Dig. 2011; 24(2):140-143. doi.org/10.1590/S0102-67202011000200010

3. Barros LM, Frota NM, Moreira RA, de Araújo TM, Caetano JÁ. Assessment of bariatric surgery results. Rev Gaucha Enferm. 2015;36(1):21-7. doi: 10.1590/1983-1447.2015.01.47694.

4. Brasil. Ministério da Saúde. Secretaria de Vigilância em Saúde. Departamento deVigilância de Doençase Agravos não Transmissíveis e Promoção da Saúde. Vigitel Brasil 2018: vigilância de fatores de risco e proteção para doenças crônicas por inquérito telefônico / Ministério daSaúde, Secretaria deVigilânciaemSaúde, Departamento de Vigilância de Doenças e Agravos não Transmissíveis e Promoção da Saúde. Brasília: Ministério da Saúde, 2019

5. Bordalo LA, Mourão DM, Bressan J. Nutritional deficiencies after bariatric surgery: why they happen?. Acta Med Port. 2011;24 Suppl 4:1021-1028. PMID: 22863513

6. Buchwald $H$, Avidor $Y$, Braunwald E, Jensen MD, Pories W, Fahrbach K, Schoelles K. Bariatric surgery: a systematic review and metaanalysis.JAMA.2004;292(14):1724-37.doi:10.1001/jama.292.14.1724. Erratum in: JAMA. 2005;293(14):1728.

7. Carvalho ADS, Rosa RDS. Bariatric surgeries performed by the Brazilian National Health System in residents of the Metropolitan Region of Porto Alegre, Rio Grande do Sul, Brazil, 2010-2016. Epidemiol Serv Saude. 2018;27(2):e2017010. doi: 10.5123/S167949742018000200008

8. Castanha CR, Tcbc-Pe ÁABF, Castanha AR, Belo GQMB, Lacerda RMR, Vilar L. Evaluation of quality of life, weight loss and comorbidities of patients undergoing bariatric surgery. Rev Col Bras Cir. 2018;45(3):e1864. doi: 10.1590/0100-6991e-20181864.

9. Conselho Federal de Medicina. Resolução CFM no 2.131/2015. Diário Oficial da União em 13 jan. 2016, Seção I, p. 66. (Retificação publicada no D.O.U. 29 jan. 2016, Seção I, p. 287

10. Conselho Federal de Medicina. Resolução CFM no 2.172/2017. Diário Oficial da União em 27 set. 2017, Seção I, p. 205.
11. Costa RC, Yamaguchi N, Santo MA, Riccioppo D, Pinto-Junior PE. Outcomes on quality of life, weight loss, and comorbidities after Roux-en-Y gastric bypass. Arq Gastroenterol. 2014;51(3):165-70. doi: 10.1590/s0004-28032014000300002.

12. da Silva PR, de Souza MR, da Silva EM, da Silva SA. Nutritional status and life quality in patients undergoing bariatric surgery. Arq Bras Cir Dig. 2014;27 Suppl 1(Suppl 1):35-8. doi: 10.1590/ s0102-6720201400s100009.

13. Goldoni MB, Fontes PRO, Guimarães MM, Diedrich-Neto JA, Nogueira T, Teixeira UF, Giacomazzi CB, Kiss G, Pioner SR, Rodrigues PD. Bypass vs. sleeve and its effects in non-alcoholic fatty liver disease: what is the best technique? Arq Bras Cir Dig. 2021;33(3):e1549. doi: 10.1590/0102-672020200003e1549.

14. Instituto Brasileiro de Geografia e Estatística. Pesquisa Nacional de Saúde:2019:atençãoprimáriaà saúdeeinformações antropométricas: Brasil / IBGE, Coordenação de Trabalho e Rendimento, [Ministério da Saúde]. [accessed in 30 de agosto de 2020]. Available in: http:www.ibge.gov.br

15. Leiro LS, Melendez-Araújo MS. Diet micronutrient adequacy of women after 1 year of gastric by-pass. Arq. Bras Cir Dig. 2014;27(Suppl.1):21-25. doi.org/10.1590/S0102-6720201400S100006

16. Lucas RWDC, Nassif PAN, Tabushi FI, Nassif DSB, Ariede BL, BritesNeto J, Malafaia O.Can stature, abdominal perimeter and bmi index predict possible cardiometabolic risks in future obesity? Arq Bras CirDig.2020;33(2):e1529. doi: 10.1590/0102-672020200002e1529.

17. Maggard $M A$, Shugarman $L R$, Suttorp $M$, Maglione $M$, Sugerman HJ, Livingston EH, Nguyen NT, Li Z, Mojica WA, Hilton L, et al. Meta-analysis: surgical treatment of obesity. Ann Intern Med. 2005;142(7):547-59. doi: 10.7326/0003-4819-142-7-20050405000013.

18. Marchesini JB, Nicareta JR. Comparative study of five different surgical techniques for the treatment of morbid obesity using BAROS. Arq Bras Cir Dig. 2014;Suppl 1(Suppl 1):17-20. doi:10.1590/ s0102-6720201400s100005.

19. Petermann-Rocha F, Durán $E$, Labraña AM, Martínez-Sanguinetti MA, Leiva AM, Garrido-Méndez A et al .What is the prevalence of metabolically healthy obesity in Chile? Rev Chil Nutr. 2019; 46(3): 264- 270. doi.org/10.4067/S0717-75182019000300264

20. Poirier $P$, Cornier MA, Mazzone T, Stiles $S$, Cummings $S$, Klein $\mathrm{S}$, McCullough PA, Ren Fielding C, Franklin BA; American Heart Association Obesity Committee of the Council on Nutrition, Physical Activity, and Metabolism. Bariatric surgery and cardiovascular risk factors: a scientific statement from the American Heart Association. Circulation. 2011;123(15):1683-701. doi: 10.1161/ CIR.0b013e3182149099.

21. Rêgo ADS, Zulin A, ScolariS, MarconSS, Radovanovic CAT. Analysis of obese patients' medical conditions in the pre and postoperative periods of bariatric surgery. Rev Col Bras Cir. 2017;44(2):171-178. doi: 10.1590/0100-69912017002011.

22. Sociedade Brasileira de Diabetes. Diretrizes da Sociedade Brasileira de Diabetes 2019-2020. (2019) 1-485 Available in: https://www. diabetes.org.br/profissionais/images/diretrizes-completa-20192020.pdf

23. Santos LA. Avaliação nutricional de pacientes obesos antes e seis meses após a cirurgia (dissertação). Belo Horizonte - MG: Universidade Federal de Minas Gerais; 2007.

24. Silva MA, Rivera IR, Barbosa EM, Crispim MA, Farias GC, Fontan AJ, Bezerra RA, Sá LG. Frequency of cardiovascular risk factors before and 6 and 12 months after bariatric surgery. Rev Assoc Med Bras (1992). 2013;59(4):381-6. doi: 10.1016/j.ramb.2013.02.009.

25. Stoll A, Silva JC, Von Bahten LC, Gugelmin G, Vedan AB, Souza Bárbara Vicente de. $O$ efeito em curto prazo do bypass gástrico sobre pacientes obesos diabéticos. Rev Col Bras Cir. 2013;40(1): 011-015. doi.org/10.1590/S0100-69912013000100003

26. World Health Organization-TheAsia-Pacific perspective:Redefining obesity and its treatment. Western Pacific Region. International Association for the Study of Obesity. Geneve: World Health Organization; 2000. 